\title{
Retinal nerve fiber layer atrophy is associated with physical and cognitive disability in multiple sclerosis
}

\author{
J Toledo', J Sepulcre , A Salinas-Alaman², A García-Layana², M Murie-Fernandez', \\ $B$ Bejarano ${ }^{1}$ and $P$ Villoslada ${ }^{1}$
}

\begin{abstract}
Background Studying axonal loss in the retina is a promising biomarker for multiple sclerosis (MS). Our aim was to compare optical coherence tomography (OCT) and Heidelberg retinal tomography (HRT) techniques to measure the thickness of the retinal nerve fiber layer (RNFL) in patients with MS, and to explore the relationship between changes in the RNFL thickness with physical and cognitive disability. We studied 52 patients with MS and 18 proportionally matched controls by performing neurological examination, neuropsychological evaluation using the Brief Repetitive Battery-Neuropsychology and RNFL thickness measurement using OCT and HRT.

Results We found that both OCT and HRT could define a reduction in the thickness of the RNFL in patients with MS compared with controls, although both measurements were weakly correlated, suggesting that they might measure different aspects of the tissue changes in MS. The degree of RNFL atrophy was correlated with cognitive disability, mainly with the symbol digit modality test $(r=0.754, P<0.001)$. Moreover, temporal quadrant RNFL atrophy measured with OCT was associated with physical disability.

Conclusion In summary, both OCT and HRT are able to detect thinning of the RNFL, but OCT seems to be the most sensitive technique to identify changes associated with MS evolution. Multiple Sclerosis 2008; 14: 906-912. http://msj.sagepub.com
\end{abstract}

Key words: cognitive impairment; Heidelberg retinal tomography; multiple sclerosis; optic coherence tomography; physical disability; retinal nerve fiber layer

\section{Introduction}

Axonal loss is the most important pathological factor that contributes to permanent disability in multiple sclerosis (MS) [1,2]. Assessing axonal loss could be useful to monitor disease evolution. Although brain atrophy measured by MRI is strongly dependent on axonal loss, it also reflects neuronal loss, synaptic pruning, loss of myelin, gliosis, and changes in water content, each of which contributes differently to permanent disability $[3,4]$. Recently, the retinal nerve fiber layer (RNFL) has been studied as a window into brain diseases [5-7], including MS [8-14]. Indeed, in a previous study we found that RNFL atrophy was correlated with disability, disease activity and brain atrophy, both at the beginning of the disease and in patients that may or may not display clinical impairment of the visual pathway [9]. Correlation between RNFL thickness and brain atrophy on the MRI have been recently confirmed $[15,16]$, indicating its ability to detect brain abnormalities. However, we still do not know if RNFL atrophy is associated with cognitive impairment as well as the different performance of the different techniques available for measuring the RNFL.

Among the different means to assess the RNFL, optical coherence tomography (OCT), Heidelberg retinal tomography (HRT) or scanning laser polarimetry (GDxVCC) focus on different aspects of the retina [17], and measurements obtained with these techniques are not equivalent [18]. OCT measurements are based on interferometry, whereby the computer analyzes the echo of reflected light from

\footnotetext{
${ }^{1}$ Department of Neurology and Neurosurgery, Clínica Universitaria de Navarra and Center for Applied Medical Research, University of Navarra, Pamplona, Spain

${ }^{2}$ Department of Ophthalmology, Clínica Universitaria de Navarra and Center for Applied Medical Research, University of Navarra, Pamplona, Spain

Correspondence to: Pablo Villoslada, Multiple Sclerosis Center, Department of Neurology, Clínica Universitaria de Navarra, 36 Pio XII, 31008 Pamplona, Spain. Email: pvilloslada@unav.es

Received 24 August 2007; revised 6 December 2007, 28 December 2007; accepted 3 February 2008
} 
a $820 \mathrm{~nm}$ laser to obtain a transverse section of the RNFL at the head of the optic nerve. By contrast, HRT investigates the optic nerve head primarily and also the retina. HRT measurements are based on obtaining different depths of two perpendicular images of the head of the optic nerve axis with a $670 \mathrm{~nm}$ laser, which are subsequently fused to obtain a three dimensional image. These technical issues indicate that OCT might better reflect axonal loss in the RNFL because it most accurately measures changes in the thickness of the tissue. Alternatively, HRT might provide more information of the overall volume changes in the thickness of the RNFL induced by different pathological process, such as the presence of edema, inflammation, changes in cell populations and axon density $[12,19,20]$. Previous studies of glaucoma and acute optic neuritis suggested that OCT is more sensitive to axonal loss and that HRT measurements, mainly the ratio of the cup to disc area, is more sensitive to volume changes in the papilla, for example those induced by inflammation or edema, in addition to axonal loss $[17,20]$. However, previous studies in glaucoma do not provide differences between OCT and GDxVCC when assessing the RNFL. However, it is not clear whether HRT measurement of the RNFL will provide additional information in patients with MS. Thus, the aim of our study was to compare the OCT and HRT techniques and to analyze the relationship between the thickness of the RNFL and physical or cognitive impairment. This cohort and data is shared with a previous study analyzing the association between RNFL, disability and MRI [9].

\section{Methods}

\section{Subjects}

We studied 52 patients with MS [21] and 18 sex and age proportionally matched healthy controls (HC). This cross-sectional study was conducted at the time of the last evaluation (month 24) in a prospective cohort used to define the association between thickness of the RNFL measured by OCT and MS activities [9]. This analysis was performed at the time that HRT became available in our centre. From the 61 patients of the original cohort, we include all patients in this study except nine patients: two were lost for follow-up and seven who did not complete both, OCT and HRT studies. We found no significant differences in demographics [age, Expanded Disability Status Scale (EDSS) or disease subtype] between lost for followup patients and the patients included in the present study. Patients were recruited by their neurologist after the study was approved by the Ethical Review Committee at the University of Navarra and having obtained written informed consent according to the Declaration of Helsinki. The inclusion criteria for patients were having been diagnosed with MS in the last 10 years, irrespective of the disease subtype. Use of immunomodulatory drugs were allowed. We excluded patients with disease duration over 10 years, with severe disability (EDSS $>7.0$ ), or with ophthalmic diseases that might impair or bias the OCT or HRT measurements (e.g. diabetes, glaucoma, congenital abnormal optic disc as tilted nerve, myopia $\geq 6$ diopters and hypermetropia $\geq 4$ diopters). Baseline neurological and ophthalmologic examinations, and OCT and HRT studies were performed in the same month.

\section{Clinical assessment}

Neurological data included number of relapses, disability progression and the use of immunomodulatory therapy recorded in a previous 2-years longitudinal study [9]. Trained staff scored disability using the EDSS, the MS Severity Scale (MSSS) and the MS Functional Composite (MSFC). We used the definition of 'disability progression' from Rio et al. [22], which proposed an increase in the EDSS in 1 point confirmed in a second visit after 6 months. Cognitive impairment was evaluated by a trained neuropsychologist with the Brief Repeatable BatteryNeuropsychology (BRB-N) as described previously [23]. Thirty-five patients performed the neuropsychological studies. Cognitive impairment was defined as a performance 1.5 SD below the control group in at least two subtests of the BRB-N. Ophthalmologic evaluations were performed by two ophthalmologists blind to the neuropsychology data and included: visual acuity (VA) using high contrast Snellen charts, direct ophthalmoscopic examination following pupil dilation using 1\% tropicamide, and the measurement of RNFL thickness by OCT and HRT. Visual impairment was defined as a best corrected VA $\leq 20 / 40$. The demographics and clinical characteristics of patients and controls, including disease activity in the previous 2 years, are shown in Table 1.

\section{Optical coherence tomography}

The thickness of the RNFL was measured in each eye using optic coherence tomography with a StratusOCT (StratusOCT 3000, and OCT 4.0 software, Carl Zeiss, Dublin) using the RNFL thickness $3.4 \mathrm{~mm}$ scan protocol after pupil dilatation. An experienced technician who was blind to the results of the other studies made the measurements. All scans were obtained three times and averaged. All scans had a signal strength requirement $>7$ (maximum 10) and involved uniform brightness across 
Table 1 Demographic and clinical variables of patients with MS and healthy controls

\begin{tabular}{lll}
\hline & $\begin{array}{l}\text { MS } \\
(\mathrm{n}=52)\end{array}$ & $\begin{array}{l}\text { Controls } \\
(\mathrm{n}=18)\end{array}$ \\
\hline Sex & $19 \mathrm{M} / 33 \mathrm{~F}$ & $7 \mathrm{M} / 11 \mathrm{~F}$ \\
Age & $37.18(8.7)$ & $34.9(8.6)$ \\
Disease duration (years) & $7.5(7.2)$ & - \\
Disease subtype & $7 \mathrm{CIS} / 36 \mathrm{RR} / 3$ & - \\
& \multicolumn{1}{c}{$\mathrm{SP} / 3 \mathrm{PP} / 4 \mathrm{PR}$} \\
Relapse rate in previous 2 years & $1.37(1.49)$ & - \\
EDSS & $2.0(0-8)$ & - \\
MSSS & $4.5(1-10)$ & - \\
MSFC & $0.114(1.06)$ & - \\
No. eyes with previous optic & 30 & - \\
$\quad$ neuritis & & - \\
No. eyes with visual impairment & 19 & \\
\hline
\end{tabular}

The results are expressed as the mean (standard deviation), except for EDSS and MSSS that it is expressed as the median and range.

M, Male; F, Female; MS, multiple sclerosis; CIS, clinically isolated syndrome; RR, relapsing-remitting; $\mathrm{SP}$, secondary-progressive; $\mathrm{PP}$, primary-progressive; PR, progressive-relapsing; EDSS, Expanded disability status scale [median (range)]; MSSS, MS severity scale [median (range)]; MSFC, MS functional composite [mean (SD)].

aVisual acuity below $0.8(20 / 40)$.

the scan circumference. The average (overall) and quadrant (temporal, superior, inferior and nasal) RNFL thickness were obtained in $\mu \mathrm{m}$. We focused our analysis in the temporal quadrant based on previous studies showing a higher reduction of the RNFL thickness in this quadrant in MS $[9,13,14]$.

\section{Heidelberg retinal tomography}

The HRT-3 scanner (Heidelberg Eye Explorer software version 1.5.1, Heidelberg Engineering, $\mathrm{GmbH}$, Heidelberg, Germany) measurements were obtained by an experimented technician, blind to the results of the other studies. Three $15^{\circ}$ topographic images were obtained for each eye and a composite image created from these three images was used for data analysis. The margin contour line of the disc to determine the disc area was drawn at the inner edge of the scleral ring by an experimented technician, assessed by an ophthalmologist in case of doubt. The software calculates various parameters, including average RNFL thickness, quadrant RNFL thickness, rim-disc area ratio, cup-disc ratio, and global rim volume. We used the cut-off point of the average RNFL minus $1.96 \mathrm{SD}$ to define a pathological RNFL using HRT [24].

\section{Statistical analysis}

The normal distribution of all variables was assessed using the Kolmogorov-Smirnov test. The differ- ences between groups were assessed with the $t$-test for parametric variables or the Mann-Whitney $U$ test for nonparametric ones. The correlation between RNFL thickness (OCT measurements were normally distributed and the HRT were not normally distributed), cognitive measures and other clinical variables was carried out using a bivariate correlation (Pearson's or Spearman's correlation). In all cases, EDSS was treated as a noncontinuous variable and analyzed with the Spearman correlation. Analysis was designed $a$ priori for testing average RNFL and temporal quadrant RNFL thickness with the clinical variables, based in our previous study [9]. We excluded eyes with previous optic neuritis and we choose one eye from every subject (patient and control) such that the optic neuritis eyes were avoided. The side (left/right) was chosen by creating a random sequence. We performed uncorrected multiple correlations for screening correlation between RNFL and cognitive measures, and we applied multiple regression analysis in variables with a $P$-value $<0.005$ (adjusted by age and education). We used the statistic package SPSS 13.0 for all analyses (SPSS Inc. Chicago, Illinois, USA).

\section{Results}

We examined the capacity of HRT to detect RNFL atrophy in MS by comparing the RNFL thickness measured in HC and patients with MS. We found that in patients with MS the average RNFL thickness and the thickness of the temporal RNFL quadrants were lower when compared with $\mathrm{HC}$, as was the global rim volume (Table 2). However, we found no differences in the other HRT variables such as the ratios of the cup to disc or rim to disc area. HRT identified RNFL atrophy in patients with a previous history of optic neuritis, either with or without permanent impairment of VA $(P=0.03$ and $P=0.011$ respectively), as well as in patients who had not previously suffered optic neuritis or any other impairment of the visual pathway $(P=0.003)$. By applying the HRT cut-off, obtained from glaucoma patients [24], we found that while the average RNFL was below the lower limit of the $95 \% \mathrm{CI}$ of the normal values in 3\% of patients with MS, none of the controls were below the cut-off. Finally, the RNFL thickness decreased in patients of all disease subtypes, including patients at the beginning of the disease such as CIS patients $(P<0.05$ in all cases). Differences between patients and controls using OCT are described elsewhere [9].

We were also interested in comparing the results of OCT and HRT measurements of the RNFL to evaluate whether these techniques are equivalent to monitor patient with MS or whether they provide complementary information about disease activity. 
Table 2 Differences in HRT variables between patients with MS and healthy controls

\begin{tabular}{lll}
\hline HRT & MS $(N=52)$ & Controls $(N=18)$ \\
\hline Average RNFL & $254.5(100.6)^{\star}$ & $275.0(49.8)$ \\
Temporal RNFL & $80(27.2)$ & $86(49.8)$ \\
Temporal inferior RNFL & $258.8(102.3)^{\star}$ & $313.8(78.1)$ \\
Temporal superior RNFL & $263.8(79.3)$ & $292.5(57.2)$ \\
Cup-disc area ratio & $0.207(0.137)$ & $0.197(0.094)$ \\
Rim-disc area ratio & $0.794(0.136)$ & $0.804(0.093)$ \\
Global rim volume & $0.368(0.140)^{\star}$ & $0.443(0.113)$ \\
\hline
\end{tabular}

The RNFL results are expressed as the mean in $\mu \mathrm{m}$ (standard deviation) and the results of the global rim volume are expressed in $\mathrm{mm}^{3}$.

HRT, Heidelberg retinal tomography; RNFL, retinal nerve fiber layer.

$* P<0.05$

We found a weak correlation between OCT and HRT RNFL thickness measures, both average and by quadrants, both in the overall cohort as well as in patients with MS (Table 3). Therefore, although both techniques provide information about the changes in the RNFL in MS patients, their measures are not equivalent and they may provide different information about tissue changes in the RNFL. We found that RNFL thickness, obtained with OCT correlated with physical disability as measured with the EDSS scale after correcting for multiple comparisons (Table 4). Moreover, patients whose disability had progressed over the previous 2 years displayed a decrease in the temporal RNFL thickness measured with OCT (Table 5). However, relapses did not appear to influence any of the HRT variables in our cohort, including the cup to disc ratio ( $P=0.139$ and $P=0.103$ respectively).

It still remains unclear whether RNFL atrophy is associated with cognitive impairment in MS. In our cohort, 16 out of 35 patients (45.7\%) displayed cognitive impairment (having a performance 1.5 SD below the control group in at least two subtests of the BRB-N), while 24 out of 35 (68.6\%) performed $1.5 \mathrm{SD}$ below the control group in at least one test. Indeed, EDSS correlates with the SRT and SDMT

Table 3 Correlation of RNFL thickness measured with OCT or HRT in MS patients

\begin{tabular}{lrlll}
\hline RNFL & \multicolumn{1}{l}{ OCT } & HRT & $r$ & $P$-value \\
\hline Average & $82.05(15.7)$ & $236(76)$ & 0.236 & 0.003 \\
Temporal & $55.42(14.4)$ & $84(27)$ & 0.281 & 0.006 \\
Nasal & $68.50(18.0)$ & $280(111)$ & 0.175 & 0.057 \\
Superior & $103.85(21.5)$ & $294(89)$ & 0.220 & 0.017 \\
Inferior & $99.91(23.4)$ & $282(107)$ & 0.191 & 0.038
\end{tabular}

Results of the RNFL thickness are expressed as the mean in $\mu \mathrm{m}$ (SD) for the MS group; and $r$ indicates the spearman correlation coefficient.

RNFL, retinal nerve fiber layer; HRT, Heidelberg retinal tomography; OCT, optic coherence tomography; SD, standard deviation.
Table 4 Correlation between RNFL thickness and physical disability

\begin{tabular}{|c|c|c|c|c|}
\hline & \multicolumn{2}{|c|}{ EDSS } & \multicolumn{2}{|c|}{ MSFC } \\
\hline & $r$ & $P$ & $r$ & $P$ \\
\hline Average RNFL OCT & -0.399 & 0.010 & 0.227 & 0.158 \\
\hline Average RNFL HRT & -0.266 & 0.093 & 0.147 & 0.373 \\
\hline Temporal RNFL OCT & -0.587 & 0.00004 & 40.440 & 0.004 \\
\hline Temporal RNFL HRT & -0.370 & 0.017 & 0.048 & 0.771 \\
\hline Temporal superior RNFL HRT & -0.144 & 0.234 & 0.158 & 0.336 \\
\hline Temporal Inferior RNFL HRT & -0.235 & 0.139 & 0.265 & 0.103 \\
\hline Cup-disc area ratio HRT & 0.241 & 0.129 & -0.255 & 0.117 \\
\hline Rim-disc area ratio HRT & -0.020 & 0.900 & -0.025 & 0.879 \\
\hline Global rim volume HRT & -0.399 & 0.010 & 0.227 & 0.158 \\
\hline
\end{tabular}

The results are shown as the correlation coefficient $(r)$ and the $P_{-}$ value. Correlation between OCT and MSFC were done using the Pearson correlation, and the other ones with Spearman correlation. Eyes with previous optic neuritis were excluded from the analysis.

HRT, Heidelberg retinal tomography; OCT, optic coherence tomography; RNFL, retinal nerve fiber layer; EDSS, expanded disability status scale; MSSS, MS Severity Scale; MSFC, MS functional composite.

$(P<0.05$; data not shown). Patients with cognitive impairment displayed a trend towards a lower average RNFL thickness when compared to patients with no cognitive impairment $(P=0.062)$. By contrast, we found that when measured by OCT, the average and temporal quadrant RNFL thickness significantly correlated with some neuropsychological test, particularly with the symbol digit modality test (SDMT) (Table 6). In the multiple regression model both OCT measures predicted SDMT values after controlling for age, and number of school years (temporal quadrant RNFL thickness adjusted $R^{2}=0.706$; and average RNFL thickness adjusted $R^{2}=0.435$ ). After including EDSS and immunomodulatory treatment in a second stepwise model, we found that correlation between temporal quadrant RNFL thickness remained significant.

\section{Discussion}

The development of new biomarkers for MS is crucial to improve our ability to predict disease evolution and monitor responses to therapy $[4,25]$. The in vivo imaging of the RNFL is a promising strategy to assess axonal loss in MS [26,27] because the visual pathway is frequently involved in this disease, retinal axons lack myelin, and it is a rapid, reproducible and relatively inexpensive technique. We and others have found that the thickness of the RNFL is diminished in patients with MS [8-14] and optic neuritis [28-31]. Moreover, the presence of RNFL atrophy is relatively accurate in predicting disease activity [9]. However, in the present study the association between RNFL thickness and disease 
Table 5 Association between RNFL thickness and disease activity

\begin{tabular}{|c|c|c|c|c|}
\hline & \multicolumn{2}{|c|}{ Relapse free } & \multicolumn{2}{|c|}{ Disability progression ${ }^{a}$} \\
\hline & Yes $(42.8 \%)$ & No $(57.2 \%)$ & No $(69 \%)$ & Yes (31\%) \\
\hline Average RNFL OCT & $87.50(13.80)$ & $76.22(14.05)^{*}$ & $83.46(13.31)$ & $76.55(17.58)$ \\
\hline Average RNFL HRT & $268.6(118.8)$ & $240.5(78.96)$ & $272.8(111.8)$ & $213.1(53.31)$ \\
\hline Temporal RNFL OCT & $58.95(11.52)$ & $54.26(14.63)$ & $60.97(12.40)$ & $46.00(9.05)^{\star \star *}$ \\
\hline Temporal superior RNFL HRT & $284.8(72.98)$ & $242.9(81.43)$ & $279.7(85.4)$ & $228.5(49.8)^{*}$ \\
\hline Rim-disc area ratio HRT & $0.791(0.14)$ & $0.797(0.14)$ & $0.802(0.13)$ & $0.775(0.15)$ \\
\hline Cup-disc area ratio HRT & $0.211(0.14)$ & $0.203(0.14)$ & $0.198(0.13)$ & $0.228(0.15)$ \\
\hline Global rim volume & $0.383(0.14)$ & $0.353(0.14)$ & $0.381(0.15)$ & $0.340(0.12)$ \\
\hline
\end{tabular}

The results of the RNFL are expressed as the mean in $\mu \mathrm{m}$ (standard deviation) and the results of the global rim volume are expressed in $\mathrm{mm}^{3}$. RNFL values were compared using the Mann-Whitney U-test.

HRT, Heidelberg retinal tomography; OCT, optic coherence tomography; RNFL, retinal nerve fiber layer.

${ }^{*} P<0.05 .{ }^{* *} P<0.01$.

${ }^{\text {aD }}$ isability progression: increase in the EDSS in 1 point confirmed in a second visit after 6 months.

activity is weak. This could be due to the fact that the RNFL is only indirectly assessing the diffuse axonal loss in the brain, and there is a lesional threshold and anatomical issue determining the contribution of axonal loss to permanent disability.

Cognitive impairment in MS is frequent and can contribute significantly to the reduced quality of life of patients with MS [23,32]. In this study, we found significant correlation between RNFL atrophy and executive and attention deficits measured with the SDMT. SDMT is the cognitive test that better identify cognitive dysfunction in MS patients and correlate with disability $[23,33]$. Thus, our results suggest that RNFL measurements are able to identify tissue changes associated with cognitive impairment. Although RNFL measurements were introduced in MS at the first time for measuring the impact of lesions in the anterior pathway (e.g. optic neuritis), previous results $[9,15]$ indicates that RNFL thickness provide an indirect estimation of brain atrophy. The ability of RNFL measurements to capture the retina counterpart of brain atrophy $[34,35]$ could explain its correlation with permanent disability and cognitive performance, independently of the involvement of the visual pathway in the functions being tested. However, several limitations might influence these findings. For example, RNFL thickness is a measure of the anterior visual pathway and it may be considered to sample the state of the brain tissue, only indirectly reflecting overall brain damage. Although the link between RNFL thickness and cognitive function may be more dependent of the general distribution of lesion load, such as more plaques in one place necessarily being associated with more plaques in others, this fact does not preclude its usefulness as a biomarker for monitoring brain damage in MS. This can explain why RNFL thickness measured with OCT have a good specificity but a low to moderate sensitivity for predicting permanent disability [9], which is highly dependent on axonal loss. These facts suggest that this technique is able to capture small changes in axonal loss, but that the patched nature of MS might prevent to reflect the overall brain damage at the retina level. In addition, our cohort was mainly composed of patients in the early- to mid-stage of the disease and cognitive impairment was not severe in the overall cohort, which might have prevented us from identifying stronger correlations with cognitive impairment.

Table 6 Correlation between RNFL thickness and cognitive impairment

\begin{tabular}{|c|c|c|c|c|c|c|c|c|c|}
\hline \multirow[t]{2}{*}{ RNFL } & \multicolumn{3}{|c|}{ Verbal memory } & \multicolumn{2}{|c|}{ Visual memory } & \multicolumn{2}{|c|}{$\begin{array}{l}\text { Executive and } \\
\text { attention }\end{array}$} & \multicolumn{2}{|c|}{ Language fluency } \\
\hline & SRT-S & SRT-R & SRT-D & SPART-S & SPART-D & SDMT & PASAT3 & WLG-p & WLG-s \\
\hline Average RNFL OCT & $0.484^{*}$ & 0.247 & 0.220 & $0.428^{*}$ & $0.466^{*}$ & $0.463^{*}$ & $0.546^{* *}$ & 0.195 & 0.214 \\
\hline Average RNFL HRT & 0.032 & 0.226 & 0.001 & 0.009 & 0.056 & 0.174 & 0.139 & -0.0323 & 0.045 \\
\hline Temporal RNFL OCT & $0.441^{*}$ & 0.272 & 0.201 & 0.289 & 0.189 & $0.754^{* * *}$ & 0.268 & 0.176 & 0.141 \\
\hline Temporal superior RNFL HRT & 0.113 & 0.205 & 0.061 & 0.174 & 0.157 & 0.175 & 0.250 & -0.120 & 0.121 \\
\hline
\end{tabular}

The results are expressed as the Spearman rank correlation.

SRT-S, Selective Reminding Test Long-Term Storage; SRT-R, Selective Reminding Test Long-Term Retrieval; SRT-D, Selective Reminding Test Delayed Recall; SPART-S, 10/36 Spatial Recall Test; SPART-D, 10/36 Spatial Recall Test Delayed; SDMT, Symbol Digit Modalities Test; PASAT 2-3, Paced Auditory Serial Addition Task 2 and $3 \mathrm{~s}$; WLG-p, Word List Generation-phonetic; WLG-s, Word List Generation-semantic.

${ }^{*} P<0.05 ;{ }^{* *} P<0.005 ;{ }^{* *} P<0.001$. 
Thus, further studies on larger cohorts and in patients with progressive disease and more severe cognitive impairment will help to better define the potential utility of RNFL measurements to assess cognitive impairment in MS.

The techniques of OCT and HRT assess structural RNFL damage in different ways [17]. OCT provides a direct measure of RNFL thickness using an algorithm capable of locating the anterior and posterior limits of the RNFL and calculating the RNFL thickness by subtraction. Conversely, HRT is an indirect measurement of RNFL thickness, extrapolated from topographic information of RNFL height and the reference plane. For this reason, HRT is more sensitive to overall volume changes in the tissue. In addition, because both techniques measured the RNFL at different location, the absolute number differs, being for OCT average RNFL in the range of the $100 \mu \mathrm{m}$ and for HRT average RNFL in the range of $250 \mu \mathrm{m}(0.25 \mathrm{~mm})$. Despite the significant difference in these techniques to measure RNFL, the thickness of the RNFL was correlated when measured by OCT and HRT in glaucomatous eyes $[17,20]$. The different technical approaches explain why previous studies in glaucoma and acute optic neuritis suggested that OCT is more sensitive to axonal loss, while HRT measurement is more sensitive to volume changes in the papilla, as produced by edema and inflammation in addition to axonal loss $[17,20]$. Because each technique is more sensitive to different tissue changes, we were interested in comparing the two techniques to assess whether they may be associated with different clinical variables, possibly reflecting the different pathogenic processes involved in MS (e.g. inflammation and axon loss). However, the fact that we did not measured the RNFL during the acute phase of optic neuritis and none of the patients had papillitis at the time of the ophthalmologic examination might prevent us from detecting the effect of inflammation in papilla volume changes.

Our study also provides valuable practical information when tempting to move RNFL measurements to neurological practice or using it as a surrogate marker for clinical trials. First, OCT seems to offer a better performance than HRT in identifying tissue changes that correlates with physical disability. Second, in addition of measuring the mean RNFL thickness, the temporal quadrant RNFL thickness measured with OCT seems to be more sensitive for detecting changes associated with disease activity. The preferential involvement of the temporal quadrant of the RNFL in MS have been described anatomically by the involvement of the maculopapillary bundle [36] and confirmed recently by OCT $[13,14]$. However, other studies have shown thinning of all RNFL quadrants using OCT in patients with optic neuritis or MS. In the study by Fisher, et al. [8], they found a decrease in all quadrants, although the decrease was smaller in the nasal quadrant. In Costello, et al. study [31], they found a decrease in all quadrants, and Trip, et al. [29] found that the superior and inferior RNFL quadrant thicknesses (but not the temporal or nasal quadrants) correlated with their corresponding visual field sectors. However, both cohorts were composed by patients with more severe ON compared with Fisher, et al. study and to our study, which might account for some loss of sensitivity. Third, by studying only eyes without previous optic neuritis we obtain a better correlate of overall brain damage. Because the presence of previous optic neuritis have a strong impact in the RNFL thickness [28$31]$, the study of the RNFL in eyes with previous optic neuritis will reflect more the local effect of the axonal loss secondary to the optic neuritis than the widespread axonal loss in the brain with MS. Further development of standards for the measurement of RNFL, similarly to the development of MRI standards for MS, as well as new software and analytical tools might increase the usefulness of this technique in MS. Moreover, a more detailed study in patients with MS with different degrees of inflammation and neurodegeneration (e.g. comparing relapsing and progressive patients with MS), and prospective studies with patients with acute optic neuritis, might clarify whether either technique has sufficient accuracy to distinguish these components.

\section{Acknowledgments}

This work was supported in part by the Spanish Ministry of Health (FIS PI051201), the 'Fundacion Uriach' and by an unrestricted grant by Gemac SA (Cenon, France) to PV. JS was a fellow of the Spanish Ministry of Health (FIS CM\#05/00222). Authors had full access to all of the data in the study and takes responsibility for the integrity of the data and the accuracy of the data analysis.

\section{References}

1. Hauser, SL, Oksenberg, JR. The neurobiology of multiple sclerosis: genes, inflammation, and neurodegeneration. Neuron 2006; 52: 61-76.

2. Imitola, J, Chitnis, T, Khoury, SJ. Insights into the molecular pathogenesis of progression in multiple sclerosis: potential implications for future therapies. Arch Neurol 2006; 63: 25-33.

3. Wegner, C, Esiri, MM, Chance, SA, Palace, J, Matthews, PM. Neocortical neuronal, synaptic, and glial loss in multiple sclerosis. Neurology 2006; 67: 960-967.

4. Miller, DH. Biomarkers and surrogate outcomes in neurodegenerative disease: lessons from multiple sclerosis. NeuroRx 2004; 1: 284-294. 
5. Miller, N, Drachman, DA. The optic nerve: a window into diseases of the brain? Neurology 2006; 67: 17421743.

6. Danesh-Meyer, HV, Birch, H, Ku, JY, Carroll, S, Gamble, G. Reduction of optic nerve fibers in patients with Alzheimer disease identified by laser imaging. Neurology 2006; 67: 1852-1854.

7. Inzelberg, R, Ramirez, JA, Nisipeanu, P, Ophir, A. Retinal nerve fiber layer thinning in Parkinson disease. Vision Res 2004; 44: 2793-2797.

8. Fisher, JB, Jacobs, DA, Markowitz, CE, et al. Relation of visual function to retinal nerve fiber layer thickness in multiple sclerosis. Ophthalmology 2006; 113: 324-332.

9. Sepulcre, J, Murie-Fernandez, M, Salinas-Alaman, A, Garcia-Layana, A, Bejarano, B, Villoslada, P. Diagnostic accuracy of retinal abnormalities in predicting disease activity in MS. Neurology 2007; 68: 1488-1494.

10. Trip, SA, Schlottmann, PG, Jones, SJ, et al. Optic nerve magnetization transfer imaging and measures of axonal loss and demyelination in optic neuritis. Mult Scler 2007; 13: 875-879.

11. Albrecht, P, Frohlich, R, Hartung, HP, Kieseier, BC, Methner, A. Optical coherence tomography measures axonal loss in multiple sclerosis independently of optic neuritis. J Neurol 2007; 254: 1595-1596.

12. Trip, SA, Schlottmann, PG, Jones, SJ, et al. Quantification of optic nerve head topography in optic neuritis: a pilot study. Br J Ophthalmol 2006; 90: 1128-1131.

13. Pulicken, M, Gordon-Lipkin, E, Balcer, LJ, Frohman, E, Cutter, G, Calabresi, PA. Optical coherence tomography and disease subtype in multiple sclerosis. Neurology 2007; 69: 2085-2092.

14. Henderson, A, Trip, S, Schlottmann, P, et al. An investigation of the retinal nerve fibre layer in progressive multiple sclerosis using optical coherence tomography. Brain 2007; 268: 12-17.

15. Gordon-Lipkin, E, Chodkowski, B, Reich, DS, et al. Retinal nerve fiber layer is associated with brain atrophy in multiple sclerosis. Neurology 2007; 69: 1603-1609.

16. Grazioli, E, Zivadinov, R, Weinstock-Guttman, B, et al. Retinal nerve fiber layer thickness is associated with brain MRI outcomes in multiple sclerosis. J Neurol Sci 2007; 131: 277-287.

17. Blumenthal, EZ, Weinreb, RN. Assessment of the retinal nerve fiber layer in clinical trials of glaucoma neuroprotection. Surv Ophthalmol 2001; 45(Suppl. 3): S305-S312.

18. Barkana, Y, Harizman, N, Gerber, Y, Liebmann, JM, Ritch, R. Measurements of optic disk size with HRT II, Stratus OCT, and funduscopy are not interchangeable. Am J Ophthalmol 2006; 142: 375-380.

19. Medeiros, FA, Zangwill, LM, Bowd, C, Weinreb, RN. Comparison of the GDx VCC scanning laser polarimeter, HRT II confocal scanning laser ophthalmoscope, and stratus OCT optical coherence tomograph for the detection of glaucoma. Arch Ophthalmol 2004; 122: 827-837.
20. Pro, MJ, Pons, ME, Liebmann, JM, et al. Imaging of the optic disc and retinal nerve fiber layer in acute optic neuritis. J Neurol Sci 2006; 250: 114-119.

21. McDonald, WI, Compston, A, Edan, G, et al. Recommended diagnostic criteria for multiple sclerosis: guidelines from the International Panel on the diagnosis of multiple sclerosis. Ann Neurol 2001; 50: 121-127.

22. Rio, J, Nos, C, Tintore, M, et al. Defining the response to interferon-beta in relapsing-remitting multiple sclerosis patients. Ann Neurol 2006; 59: 344-352.

23. Sepulcre, J, Vanotti, S, Hernandez, R, et al. Cognitive impairment in patients with multiple sclerosis using the Brief Repeatable Battery-Neuropsychology test. Mult Scler 2006; 12: 187-195.

24. Wollstein, G, Garway-Heath, DF, Hitchings, RA. Identification of early glaucoma cases with the scanning laser ophthalmoscope. Ophthalmology 1998; 105: 1557-1563.

25. Bielekova, B, Martin, R. Development of biomarkers in multiple sclerosis. Brain 2004; 127: 1463-1478.

26. Frohman, EM, Costello, F, Zivadinov, R, et al. Optical coherence tomography in multiple sclerosis. Lancet Neurol 2006; 5: 853-863.

27. Sergott, RC, Frohman, E, Glanzman, R, Al-Sabbagh, A. The role of optical coherence tomography in multiple sclerosis: expert panel consensus. J Neurol Sci 2007; 263: 3-14.

28. Parisi, V, Manni, G, Spadaro, M, et al. Correlation between morphological and functional retinal impairment in multiple sclerosis patients. Invest Ophthalmol Vis Sci 1999; 40: 2520-2527.

29. Trip, SA, Schlottmann, PG, Jones, SJ, et al. Retinal nerve fiber layer axonal loss and visual dysfunction in optic neuritis. Ann Neurol 2005; 58: 383-391.

30. Trip, SA, Schlottmann, PG, Jones, SJ, et al. Optic nerve atrophy and retinal nerve fibre layer thinning following optic neuritis: evidence that axonal loss is a substrate of MRI-detected atrophy. Neuroimage 2006; 31: 286-293.

31. Costello, F, Coupland, S, Hodge, W, et al. Quantifying axonal loss after optic neuritis with optical coherence tomography. Ann Neurol 2006; 59: 963-969.

32. Amato, MP, Zipoli, V, Portaccio, E. Multiple sclerosisrelated cognitive changes: a review of cross-sectional and longitudinal studies. J Neurol Sci 2006; 245: 41-46.

33. Parmenter, BA, Weinstock-Guttman, B, Garg, N, Munschauer, F, Benedict, RH. Screening for cognitive impairment in multiple sclerosis using the Symbol digit Modalities Test. Mult Scler 2007; 13: 52-57.

34. Frohman, E, Zivadinov, R. What you see is what you get: coupling function with structure in the visual system. Neurology 2007; 69: 2119-2120.

35. Wu, GF, Schwartz, ED, Lei, T, et al. Relation of vision to global and regional brain MRI in multiple sclerosis. Neurology 2007; 69: 2128-2135.

36. Kerrison, J, Flynn, T, Green, W. Retinal pathologic changes in multiple sclerosis. Retina 1994; 14: 445-451. 